\title{
The PRolaCT studies - a study protocol for a combined randomised clinical trial and observational cohort study design in prolactinoma
}

Ingrid M. Zandbergen ${ }^{1,2,3^{*}}$ (D), Amir H. Zamanipoor Najafabadi ${ }^{1,3}$, Iris C. M. Pelsma ${ }^{2,3}$, M. Elske van den Akker-van Marle ${ }^{4}$, Peter H. L. T. Bisschop ${ }^{5}$, H. D. Jeroen Boogaarts ${ }^{6}$, Arianne C. van Bon ${ }^{7}$, Bakhtyar Burhani ${ }^{8}$, Saskia le Cessie ${ }^{4,9}$, Olaf M. Dekkers ${ }^{2,9}$, Madeleine L. Drent ${ }^{10}$, Richard A. Feelders ${ }^{11}$, Johan P. de Graaf ${ }^{12}$, J. Hoogmoed ${ }^{13}$, Kitty K. Kapiteijn ${ }^{14}$, Melanie M. van der Klauw ${ }^{15}$, Willy-Anne C. M. Nieuwlaat ${ }^{16}$, Alberto M. Pereira ${ }^{2,3}$, Aline M. E. Stades ${ }^{17}$, Annenienke C. van de Ven ${ }^{18}$, Iris M. M. J. Wakelkamp ${ }^{19}$, Wouter R. van Furth ${ }^{1,3}$, Nienke R. Biermasz $z^{2,3}$ and on behalf of the Dutch Prolactinoma Study Group

\begin{abstract}
Background: First-line treatment for prolactinomas is a medical treatment with dopamine agonists (DAs), which effectively control hyperprolactinaemia in most patients, although post-withdrawal remission rates are approximately 34\%. Therefore, many patients require prolonged DA treatment, while side effects negatively impact health-related quality of life (HRQoL). Endoscopic transsphenoidal resection is reserved for patients with severe side effects, or with DA-resistant prolactinoma. Surgery has a good safety profile and high probability of remission and may thus deserve a more prominent place in prolactinoma treatment. The hypothesis for this study is that early or upfront surgical resection is superior to DA treatment both in terms of HRQoL and remission rate in patients with a non-invasive prolactinoma of limited size.

Methods: We present a combined randomised clinical trial and observational cohort study design, which comprises three unblinded randomised controlled trials (RCTs; PRolaCT-1, PRolaCT-2, PRolaCT-3), and an observational study arm (PRolaCT-O) that compare neurosurgical counselling, and potential subsequent endoscopic transsphenoidal adenoma resection, with current standard care. Patients with a non-invasive prolactinoma $(<25$ $\mathrm{mm}$ ) will be eligible for one of three RCTs based on the duration of pre-treatment with DAs: PRolaCT-1: newly diagnosed, treatment-naïve patients; PRolaCT-2: patients with limited duration of DA treatment (4-6 months); and PRolaCT-3: patients with persisting prolactinoma after DA treatment for $>2$ years. PRolaCT-O will include patients who decline randomisation, due to e.g. a clear treatment preference. Primary outcomes are disease remission after 36 months and HRQoL after 12 months.
\end{abstract}

\footnotetext{
* Correspondence: prolactinoom@lumc.nl

'Department of Neurosurgery, Leiden University Medical Center, Albinusdreef 2, 2333ZA Leiden, The Netherlands

2Department of Medicine, Division of Endocrinology, Leiden University Medical Center, Albinusdreef 2, 2333ZA Leiden, The Netherlands

Full list of author information is available at the end of the article
}

(c) The Author(s). 2021 Open Access This article is licensed under a Creative Commons Attribution 4.0 International License, which permits use, sharing, adaptation, distribution and reproduction in any medium or format, as long as you give appropriate credit to the original author(s) and the source, provide a link to the Creative Commons licence, and indicate if changes were made. The images or other third party material in this article are included in the article's Creative Commons licence, unless indicated otherwise in a credit line to the material. If material is not included in the article's Creative Commons licence and your intended use is not permitted by statutory regulation or exceeds the permitted use, you will need to obtain permission directly from the copyright holder. To view a copy of this licence, visit http://creativecommons.org/licenses/by/4.0/ The Creative Commons Public Domain Dedication waiver (http://creativecommons.org/publicdomain/zero/1.0/) applies to the data made available in this article, unless otherwise stated in a credit line to the data. 
Discussion: Early or upfront surgical resection for patients with a limited-sized prolactinoma may be a reasonable alternative to the current standard practice of DA treatment, which we will investigate in three RCTs and an observational cohort study. Within the three RCTs, patients will be randomised between neurosurgical counselling and standard care. The observational study arm will recruit patients who refuse randomisation and have a pronounced treatment preference. PRolaCT will collect randomised and observational data, which may facilitate a more individually tailored practice of evidence-based medicine.

Trial registration: US National Library of Medicine registry (ClinicalTrials.gov) NCT04107480. Registered on 27 September 2019, registered retrospectively (by 2 months).

Keywords: Prolactinoma, Pituitary tumour, Dopamine agonist, Endoscopic transsphenoidal resection, Randomised clinical trial, Observational cohort

\section{Administrative information}

Trial registration

Primary identifying number:

CCMO-Registry ID: NL63919.058.18

Secondary identifying numbers:

ZonMw Grant ID: 843002806

ClinicalTrials.gov ID: NCT04107480

Protocol version: 5

Issue date: 7 Jan 2021

Protocol amendment number: 3

Trials Sponsor (coordinating centre): Leiden University Medical Center

\section{Background}

Prolactin-producing pituitary adenomas typically cause functional hyperprolactinaemia, which is characterised by galactorrhoea and hypogonadism, and may therefore result in subfertility [1]. Additionally, non-specific symptoms, such as fatigue, and psychological and neurocognitive complaints have been described [1]. Although a rare condition, prolactinomas account for over half (32-66\%) of all pituitary adenomas [1]. Prolactinomas are found 3 times more frequent in women than in men with a peak incidence between the age of 25 and 35 years $[2,3]$. The majority of prolactinomas are subcategorised, based on the size on magnetic resonance imaging (MRI), as microprolactinomas $(<10 \mathrm{~mm} ; 80 \%)$, but they may also present as macro $(10-40 \mathrm{~mm})$ or giant $(>40 \mathrm{~mm})$ and invasive tumours, which may compromise visual function due to compression of the optic chiasm, nerves, or tracts [2].

Prolactinoma treatment is primarily targeted at reduction of symptoms and restoration of gonadal status and fertility by normalising prolactin levels [4]. As it is a highly effective and non-invasive treatment option, medical treatment with a dopamine agonist (DA) is recommended as first-line treatment for almost all prolactinoma patients, while endoscopic transsphenoidal resection is reserved for those patients with (severe) DA intolerance or in case of DA-resistant prolactinoma [4].
Although DA treatment results in normalisation of prolactin levels, and subsequent reduction of symptoms in $81 \%$ of patients, the 2 -year remission rate after DA withdrawal remains low (34\%) and, therefore, long-term medical treatment should be expected [5]. DA treatment is usually well tolerated, although orthostatic hypotension and gastrointestinal complaints are frequently reported as side effects. DAs are furthermore associated with psychological side effects, which are mild in $40 \%$, but more severe in up to $5-10 \%$ of patients [5-13]. Particularly, impulse control disorders (ICDs), e.g. pathologic gambling, are acknowledged as a typical side effect [1316]. The heterogenous pattern of side effects and prolactinoma symptomatology may (in part) explain that both untreated (symptomatic) and medically treated prolactinoma patients have reported a decreased physical and mental health-related quality of life (HRQoL) [5, 17-22].

Although clearly more invasive, endoscopic pituitary surgery has a well-established good safety profile for prolactinomas of limited size. Long-term morbidity from complications occurs in less than $2-3 \%$ of patients (mostly due to hypopituitarism or permanent diabetes insipidus), whereas transient perioperative complications, such as cerebrospinal fluid leakage and transient diabetes insipidus, occur in less than 5 and $15 \%$ of patients, when surgery is performed by experienced neurosurgeons [5, 17, 23-27]. Resulting in immediate cure in 80 to $90 \%$ of prolactinoma patients, endoscopic transsphenoidal surgery is also a highly effective treatment [5, 17, 23-25]. Moreover, the vast majority of postoperative patients will not need long-term DA treatment, since recurrence rates after surgery are $4-15 \%[5,17,28]$. Interestingly, two recent simulation studies have estimated that endoscopic surgery could be a more cost-effective treatment when compared to cabergoline, the most effective DA $[29,30]$.

With improvement of the endoscopic transsphenoidal surgical technique, increasing evidence of potentially 
detrimental side effects of DAs, and disappointing remission rates after a period of at least 2 years of DA treatment, equipoise may exist for these treatment options, particularly for patients with the greatest likelihood for successful complete adenoma resection (i.e. clearly visible, of limited size, and non-invasive tumours). Nevertheless, the efficacy-to-safety ratios of surgery and medical treatment are highly different and incomparable, so HRQoL after surgery (without DA in most cases) and risk of complications should be weighed against HRQoL during (often long-term) medical treatment and its potential side effects. However, there are currently only a handful of retrospective observational studies published on this topic with major drawbacks, such as selection bias and insufficient and inappropriate correction for confounding variables [5]. Therefore, we believe that this is the right moment in time to perform a randomised controlled trial (RCT) comparing both treatment strategies.

We hypothesise that endoscopic transsphenoidal adenoma resection as a first-line, or equally valid second-line, treatment is superior to standard care (DA treatment) for patients with a visible prolactinoma of limited size $(<$ $25 \mathrm{~mm}$, no tissue invasion). The primary outcome parameters are (1) HRQoL measured after 12 months of follow-up (i.e. 12 months after randomisation or, in PRolaCT-O, study baseline) and (2) the proportion of patients in remission at 36 months of follow-up/study baseline.

\section{Methods}

\section{Study design}

This combined randomised clinical trial and observational cohort study design comprises three unblinded parallel superiority prolactinoma RCTs (PRolaCT-1, PRolaCT-2, PRolaCT-3) and an observational study arm (PRolaCT-O) that will compare counselling for endoscopic transsphenoidal adenoma resection to standard care (DA treatment) in patients with a prolactinoma of limited size (i.e. $<25 \mathrm{~mm}$, no tissue invasion):

1. PRolaCT-1 compares counselling for surgical resection as a first-line treatment in newly diagnosed, treatment-naïve prolactinoma patients.

2. PRolaCT-2 compares counselling for surgical resection as an early second-line treatment in patients who have had DA treatment for a limited time period (4-6 months).

3. PRolaCT-3 compares counselling for surgical resection as an equal second-line treatment in patients who have persisting prolactinoma, i.e. recurring hyperprolactinaemia and persisting pituitary adenoma on MRI, after DA treatment for a long period of time ( $>2$ years).
4. PRolaCT-O evaluates counselling for surgical resection as an equally valid second-line treatment and standard care in an observational study setting, aiming to include all patients eligible for PRolaCT1, PRolaCT-2, and PRolaCT-3 who do not participate in the RCTs because of a strong patient and/or physician treatment preference, and/or because the patient does not consent to randomisation.

All interventions and study procedures are otherwise identical for the three RCTs and the observational cohort.

Since some patients will be unwilling to be randomised for various reasons, such as a strong preference for either surgery or DA treatment, there is a risk that for the RCT a selected subpopulation is recruited, excluding patients with more pronounced side effects of DA treatment or those with an adenoma that is expected to be relatively difficult to resect completely. Especially outcomes in HRQoL might be attenuated or overestimated and there is a risk that the results cannot be generalised to all prolactinoma patients. The PRolaCT-O observational arm was added to provide a fall-back mechanism for evaluation of primary and secondary study objectives in case recruitment would prove to be challenging and to provide data for the evaluation of a possible selection bias in the RCTs, and is designed to collect real-life data about the eligible cohort. The combined RCT and observational cohort approach was received well by our advisory board (including a patient representative) and better reflects clinical decision-making in real life, as the MDT or treating physician may also have a preference due to tumour- and patient-specific characteristics. Furthermore, the prospective data collection of PRolaCT-O includes comprehensive information about expected confounders, such as duration and effectiveness of prior DA treatment, the amount and severity of side effects of DA treatment, and expected difficulty of surgery as judged by two blinded neurosurgeons.

\section{Study setting}

Endoscopic surgery is centralised in a few tertiary expert neurosurgical centres, while endocrine care for prolactinoma is delivered by endocrinologists and gynaecologists in all hospitals. For an expected enrolment period of 3 years, starting June 2019, recruitment for PRolaCT operates from five participating neurosurgical reference centres and uses regional multidisciplinary networks to recruit patients from the referring hospitals. PRolaCT is furthermore intended to expand to other reference centres in the Netherlands and internationally to the UK, France, and Germany, in collaboration with European Networks. 


\section{Study population}

Eligibility to participate with one of the RCTs or PRolaCT-O is based on the following inclusion and exclusion criteria.

\section{Inclusion criteria}

- At least 18 years of age

- A history of signs and symptoms matching with prolactinoma

- Hyperprolactinaemia, defined as a prolactin level $\geq 2$ times the local laboratory maximum, present at the time of enrolment (PRolaCT-1) or present $<12$ months before enrolment (PRolaCT-2 and PRolaCT-3)

- No clear alternative explanation for hyperprolactinaemia, e.g. medication use

- Presence of a clearly identifiable pituitary mass on MRI not invading the cavernous sinus and with a maximum diameter $\leq 25 \mathrm{~mm}$. A representative MRI at the time of randomisation is required. This MRI should generally not be older than 12 months in PRolaCT-3 and 2 months in PRolaCT-1 and PRolaCT-2

- Defining inclusion criteria for the three RCTs are:

- PRolaCT-1: no prior prolactinoma treatment

- PRolaCT-2: prior DA treatment for 4-6 months or

- PRolaCT-3: prior DA treatment for at least 2 years and having conducted at least one withdrawal attempt within 12 months prior to study recruitment (if the withdrawal attempt took place $>6$ months before study inclusion, another withdrawal attempt is performed before randomisation to confirm prolactinoma persistence)

\section{Exclusion criteria}

- Contraindication for one of the treatment modalities, e.g. severe side effects of DA treatment or DA-resistant prolactinoma

- Contraindications to surgery, or a clear indication for surgical resection

- Pregnancy at the time of randomisation

- Clinical diagnosis of acromegaly

- Prior radiotherapy of the pituitary gland area

- Severe renal failure (eGFR $<30 \mathrm{ml} / \mathrm{min}$ )

- Insufficient understanding of the Dutch and English language

Patients eligible for participation in one of the RCTs, but do not consent to randomisation because there is a clear patient preference for either DA treatment or surgery, are considered for participation in PRolaCT-O, also see Fig. 1.

All prolactinoma patients are screened for eligibility to participate with one of the RCTs. Patients who decline randomisation or have a strong treatment preference are approached for PRolaCT-O. All ineligible patients will be approached for ProlaC, an additional registry including all prolactinoma patients (e.g. invasive tumours, pregnant patients).

\section{Patient identification and enrolment}

Eligible patients are discussed within a regional multidisciplinary network, operating from the participating neurosurgical expertise centres. RCT eligibility is confirmed by the regional MDT. The counselling of new, eligible patients will be performed in the following order: first, willingness to participate in the RCT (PRolaCT-1, 2,3 ) will be assessed, followed by willingness to be included in the intensive observational cohort with shared decision-making (PRolaCT-O). Patients who fulfil RCT eligibility but do not consent to randomisation or have a clear preference for standard care (PRolaCT-1) or either treatment modality (PRolaCT-2 and PRolaCT-3) are approached for participation in the observational arm, PRolaCT-O, which is depicted in Fig. 1. Patients who were approached for PRolaCT-1, but do not participate with PRolaCT, may be approached again for participation in PRolaCT-2 or PRolaCT-3, when the duration of DA treatment fulfils the inclusion criteria of the respective RCT. Likewise, patients who are enrolled in PRolaCT-O and receive standard care may be approached for participation in PRolaCT-2 or PRolaCT3 if they reconsider surgical treatment. Written informed consent will be obtained from all participants prior to study inclusion.

\section{Treatment allocation}

Within each RCT (PRolaCT-1, PRolaCT-2, or PRolaCT3 ), participants are randomly allocated in a 1:1 ratio to either neurosurgical counselling (with potential subsequent surgery when desired) or standard care with DA. Randomisation is performed in blocks of sizes varying between 4,6 , and 8 patients and is stratified by sex to equally divide male participants and by tumour size $(<$ $10 \mathrm{~mm}$ versus $\geq 10 \mathrm{~mm}$ ). Randomisation is performed centrally in the LUMC using the built-in randomisation tool of the web-based database programme (Castor Electronic Data Capture (EDC), Castor, Amsterdam, the Netherlands). Investigators, treating physicians, and participants will not be blinded to the outcomes of randomization. With the use of varying block sizes, the allocation sequence is kept concealed by the randomisation programme. In PRolaCT-O, treatment choice, based on patient and physician preference, is solely recorded 


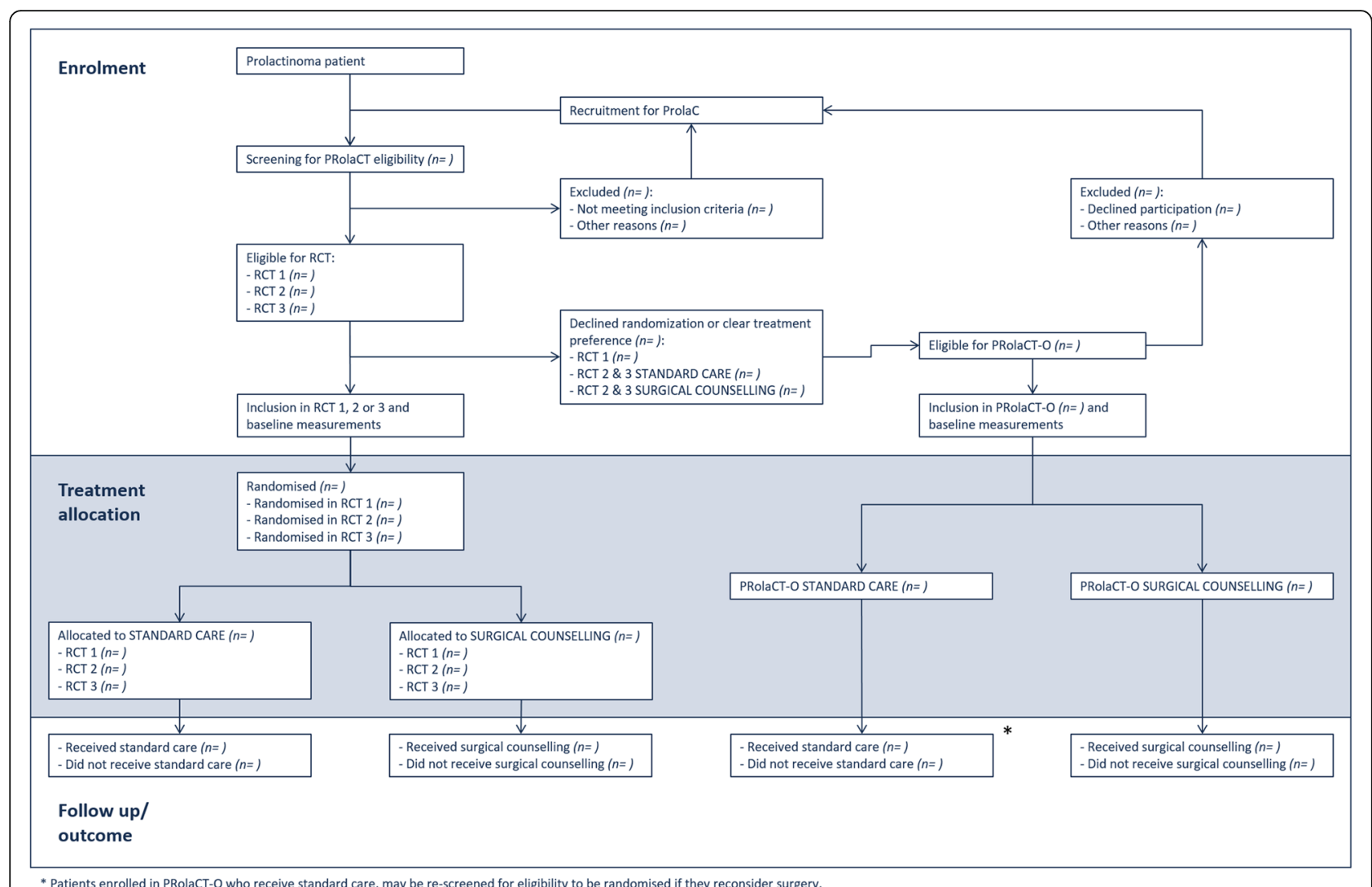

Fig. 1 Overview of patient recruitment and treatment allocation

and may be DA treatment as a first- or second-line treatment, or surgical counselling as a second-line treatment (thus not in newly diagnosed patients). For all studies, the interpreters of MRIs, ophthalmologic assessments, and surgical difficulty will be blinded when possible. Unblinding will not occur.

\section{Treatment}

\section{Intervention: surgical counselling}

Participants who are randomised for the intervention group will receive extensive and personalised neurosurgical counselling by a neurosurgeon and an endocrinologist with specific expertise in pituitary care (preferably a combined consultation). All potential benefits and risks of surgery are discussed in a semi-standardised manner, offering both standardised information on surgical intervention and detailed expert opinion regarding the patient's specific situation. This consultation may theoretically result in advising the patient to refrain from surgery during the shared decision-making process, despite the patient's previous eligibility "on paper". Moreover, based on information gathered during the counselling, the MDT may advise against surgery and propose standard treatment as a better alternative in this individualised approach. After counselling, the patient is asked for consent for surgery as is a standard preoperative requirement regardless of study participation. Participants in the intervention group may thus decide not to proceed with the surgical intervention. Participants who do not consent to surgery will continue the RCT in the intervention group, but receive treatment according to standard care. Patients receiving standard of care may be referred back to their own physician following the counselling.

When a participant and the MDT agree to surgery, an endoscopic transsphenoidal surgical resection of the prolactinoma is performed according to standard practice in an expertise centre. Patients are hospitalised for 2-5 days postoperatively. In the first weeks after discharge from the hospital, frequent contact by phone or visits to the outpatient clinic are needed to check for complications and recovery of the patient, according to the standards of care of the neurosurgical centre. The frequency of these visits is gradually reduced to (half-)yearly visits depending on the patient's well-being.

\section{Control: standard care}

All participants who are randomised for standard care will receive treatment as usual from their own 
endocrinologist or gynaecologist as described by the US Endocrine Society [4].

First-line treatment is with DA, of which cabergoline is used most often; alternatives are bromocriptine and quinagolide. The dosage is usually titrated to achieve a normal or suppressed prolactin level in combination with restoration of the gonadal axis. DA treatment is discontinued after 2 years of treatment, i.e. 24 months after randomisation, unless a normal prolactin level cannot be achieved, and is restarted when prolactin levels rise after the medication is discontinued. As is customary in standard care, patients in the control group may be offered surgery in case of DA intolerance (side effects) or an insufficient response to DA treatment.

All participants may use any form of co-medication when medically indicated. Surgery is not performed on a pregnant participant, unless specifically indicated. Women who are pregnant at the time of randomisation are therefore not eligible for inclusion in an RCT. If a patient enrolled in one of the RCTs becomes pregnant after randomisation, she will remain in the trial, but treatment will be adapted, according to standard clinical care.

Our intervention protocol has been conceived in agreement with current standards of care for prolactinoma patients with an indication for surgery. Therefore, there are no specific strategies to improve adherence to intervention protocols. However, as part of the outcome data, we do monitor which treatment, surgical or medical, participants receive.

\section{Outcome assessment}

Assessment of clinical outcomes takes place during outpatient clinic visits at 12, 27, 36, and 60 months after randomisation (PRolaCT-1, PRolaCT-2, and PRolaCT3 ), or after study baseline (PRolaCT-O). For PRolaCT-O, study baseline is defined as the moment randomisation would have taken place, thus after all baseline measurements are performed and when treatment is chosen. An extensive overview of enrolment, interventions, and outcome assessment is given in Table 1. During these visits to the outpatient clinic, registration of prolactinoma treatment, physical symptoms, pregnancy, laboratory measurements (including prolactin level), and pituitary MRI (only at 12 and 36 months) takes place. For all surgically treated patients, the final registration of complications takes place during the outpatient clinic visit at 12 months. At every time point, assessment of patientreported outcome measurements (PROMs) will take place using self-reported questionnaires. These questionnaires assess HRQoL and the presence and burden of physical and psychiatric symptoms or side effects to treatment. In all PRolaCT study arms, PROMs will additionally be collected every 3-6 months after randomisation/study baseline until $T=36$ for a costeffectiveness analysis.

\section{Primary outcomes}

The two primary outcomes PRolaCT aims to evaluate are:

1. Health-related quality of life after 12 months of follow-up

2. Disease remission after 36 months of follow-up

\section{Health-related quality of life}

The primary outcome measure HRQoL is defined as the score on the mental health scale (MHS) of the Medical Outcomes Study (MOS) Short-Form Health Survey (SF36) after 12 months of follow-up, a time point reflecting a stable situation after surgical treatment or during DA treatment. The SF-36 is the most frequently used questionnaire in prolactinoma patients and has been validated in Dutch and English [22, 31]. The SF-36 is composed of 36 items, organised into eight multi-item scales, including the MHS. The seven other scales assess physical functioning, role limitations due to physical health problems, bodily pain, general health perceptions, vitality, social functioning, and role limitations due to emotional problems [31-33]. In addition to scoring on these scales, the SF-36 also yields two higher order component scores, one for physical health and one for mental health [31-33]. For our primary endpoint, only the score on the MHS will be used, and scores on the other scales and the two component scores will be used for secondary outcome measurements. The MHS score ranges from 0 to 100 and is known to be decreased in patients with active or treated prolactinomas [18-22].

\section{Disease remission}

Disease remission is defined as the presence of either normoprolactinaemia, which is defined as a prolactin level below the upper limit of normal for the respective laboratory, in the absence of DA treatment for at least 3 months; or pregnancy that was established during at least 3 months of withdrawal of DA treatment. The primary outcome assessment of disease remission is at 36 months of follow-up, reflecting remission after 2 years of DA treatment or long-term remission after surgery.

\section{Secondary outcomes}

The secondary outcomes for this study will be:

- HRQoL measured with the SF-36 (other than the MHS) at baseline and 12, 36, and 60 months after randomisation/baseline

- Disease remission 27 and 60 months after randomisation/baseline 
Table 1 Overview of enrolment, interventions, and outcome assessment

\begin{tabular}{|c|c|c|c|c|c|c|c|c|}
\hline \multirow[b]{2}{*}{ TIME POINTS } & \multirow{2}{*}{$\begin{array}{c}\text { Enrolment } \\
\mathrm{T}=\mathrm{X}\end{array}$} & \multirow{2}{*}{$\begin{array}{c}\text { Allocation } \\
\mathrm{T}=0\end{array}$} & \multicolumn{6}{|c|}{ Post-allocation } \\
\hline & & & $<3$ months & $\mathrm{T}=12$ & 24 months & $\mathrm{T}=27$ & $\mathrm{~T}=36$ & $\mathrm{~T}=60$ \\
\hline \multicolumn{9}{|l|}{ ENROLMENT: } \\
\hline \multirow{2}{*}{$\begin{array}{l}\text { Eligibility screening } \\
\text { Informed consent }\end{array}$} & $x$ & & & & & & & \\
\hline & $x$ & & & & & & & \\
\hline \multirow{2}{*}{$\begin{array}{c}\text { Baseline measurements } \\
\text { Treatment allocation }\end{array}$} & $x$ & & & & & & & \\
\hline & & $\mathrm{X}$ & & & & & & \\
\hline \multicolumn{9}{|l|}{ INTERVENTIONS: } \\
\hline \multicolumn{9}{|l|}{ Intervention groups: } \\
\hline \multicolumn{9}{|l|}{ Surgical counselling } \\
\hline \multicolumn{9}{|l|}{ Surgery } \\
\hline \multicolumn{9}{|l|}{ Standard care groups: } \\
\hline \multicolumn{9}{|l|}{ DA treatment } \\
\hline \multicolumn{9}{|l|}{ Withdrawal attempt } \\
\hline \multirow{4}{*}{$\begin{array}{r}\text { ASSESSMENTS: } \\
\text { Prolactinoma treatment } \\
\text { Physical symptoms } \\
\text { Pregnancy (when applicable) }\end{array}$} & $\mathrm{x}$ & & & & & & & \\
\hline & $\mathrm{X}$ & & & $x$ & & $x$ & 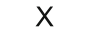 & $\mathrm{X}$ \\
\hline & $\mathrm{X}$ & & & $x$ & & $x$ & $X$ & $\mathrm{X}$ \\
\hline & $\mathrm{X}$ & & & $x$ & & $x$ & $\mathrm{X}$ & $x$ \\
\hline \multirow{4}{*}{$\begin{array}{r}\text { Surgical complications } \\
\text { Side effects of dopamine agonist } \\
\text { Prolactin measurement } \\
\text { Other pituitary axes }\end{array}$} & & & & $\mathrm{x}$ & & & & \\
\hline & $\mathrm{X}$ & & & $x$ & & $x$ & $\mathrm{X}$ & $x$ \\
\hline & $X$ & & & $x$ & & $x$ & $\mathrm{X}$ & $\mathrm{X}$ \\
\hline & $\mathrm{X}$ & & & $x$ & & & $x$ & \\
\hline MRI & $x$ & & & $x$ & & & $x$ & \\
\hline PRO-CTCAE/HADS & $x$ & & & $\mathrm{X}$ & & $x$ & $x$ & $\mathrm{x}$ \\
\hline SF-36 & $\mathrm{X}$ & & & $x$ & & $x$ & $\mathrm{X}$ & $\mathrm{X}$ \\
\hline LBNQ & $\mathrm{X}$ & & & $x$ & & & $\mathrm{X}$ & \\
\hline$E Q-5 D-5 L^{a}$ & $x$ & & & & & & & $x$ \\
\hline \multicolumn{9}{|l|}{$\mathrm{iMCQ}^{\mathrm{b}}$} \\
\hline \multicolumn{9}{|l|}{$\mathrm{iPCQ}^{\mathrm{b}}$} \\
\hline \multirow{2}{*}{$\begin{array}{l}\text { ICD Questionnaire } \\
\text { Treatment Motivation }^{c}\end{array}$} & $x$ & & & $x$ & & $x$ & $\mathrm{X}$ & \\
\hline & $\mathrm{X}$ & & & & & & & \\
\hline
\end{tabular}

${ }^{a}$ Measured 6, 9, 12, 18, 24, 27, 30, and 36 months after $T=0$

${ }^{b}$ Measured every 6 months after $T=0$

'Only in PRolaCT-O

- Biochemical disease control, defined as a normalised prolactin or actual pregnancy 12 months after randomisation/baseline

- Recurrence rate defined as recurrence of hyperprolactinaemia after achieving disease remission, measured 36 and 60 months after randomisation/baseline in all patients who were in remission at the 27-month follow-up time point

- Clinical symptom control, defined as the absence of clinical symptoms, registered in patient's medical records and measured with the use of the National Cancer Institute Patient-Reported Outcomes version 
of the Common Terminology Criteria for Adverse Events (PRO-CTCAE) at 12, 27, 36, and 60 months after randomisation/baseline

- Tumour shrinkage on MRI 12 and 36 months after randomisation/baseline

- Pituitary functioning 12, 36, and 60 months after randomisation/baseline

- Complications of surgery registered in the patient's medical records or side effects to DA treatment, measured with the PRO-CTCAE and a modified Impulse Control Disorder Questionnaire (ICD-Q), 12 and 36 months after randomisation/baseline

- Depression and anxiety scores, measured with the Hospital Anxiety and Depression Scale (HADS), 12 and 36 months after randomisation/baseline

- Disease burden, measured with the Leiden Bother and Needs Questionnaire (LBNQ), 12, 36, and 60 months after randomisation/baseline

- Cost-effectiveness at 12 and 36 months after randomisation/baseline measured with the EQ-5D$5 \mathrm{~L} 6,9,12,18,24,27,30$, and 36 months after randomisation/baseline and the iMTA Productivity Cost Questionnaire (iPCQ) and the iMTA Medical Consumption Questionnaire (iMCQ) once every 6 months, starting 6 months after randomisation/baseline, until $T=36$

\section{Sample size calculation}

To detect a difference in remission rate at 36 months of $40 \%$ between surgery (expected rate $90 \%$ ) and medical treatment (expected rate max. 50\%), with a power of 0.8 and an $\alpha$ of $0.025,55$ patients per treatment group arm are needed, when considering that max. $30 \%$ in the interventional arm will not undergo surgery. However, to detect a difference of 10 points at the SF-36 mental health scale at $T=12$ with an $\alpha$ of 0.025 and a power of 0.8 , assuming that the standard deviation is 16 , based on the general population [31], and that max. $30 \%$ of the interventional arm does not undergo surgery, at least 101 patients per treatment arm are needed. To compensate for the loss to follow-up, it is aimed to include 110 patients per treatment arm and therefore 220 patients in total per RCT/PRolaCT-O (aiming for at least 110 patients per treatment group in PRolaCT-O). If patient recruitment would prove challenging, and the required sample size for separate analyses of the RCTs cannot be reached for two or more of the RCTs (PRolaCT-1, PRolaCT-2, or PRolaCT-3) within the duration of the study, the planned analyses could be performed using the combined sample size for two to three RCTs of 220 patients.

\section{Planned statistical analyses}

We intend to perform all statistical analyses separately for PRolaCT-O and PRolaCT-1, PRolaCT-2, and
PRolaCT-3. However, a combined analysis of two to three RCTs may be performed, as stated above, using a regression analysis with adjustment for randomisation group type. A post hoc meta-analysis including all three RCTs and PRolaCT-O will be performed, whatever the course of the study.

To account for positive results due to multiple testing, a lower than usual $p$-value, i.e. $<0.02$, will be considered statistically significant. All statistical analyses will be performed according to the intention-to-treat principle. In a sensitivity approach, a per-protocol analysis will also be performed, which may be of added value in case many patients, after consultation, decide not to be operated.

\section{Main endpoints}

Health-related quality of life In the three RCTs, the difference between the two treatment arms in MHS of the SF-36 will be compared at 12 months using a linear regression analysis, using treatment and the baseline measurement as covariates (analysis of covariance).

Disease remission In the three RCTs, the percentage of patients that achieve disease remission at 36 months will be calculated for the two treatment arms with corresponding 95\% confidence interval and compared using a chi-square test.

Analyses in PRolaCT-O using propensity score methods As the treatment groups in PRolaCT-O may differ at baseline, propensity score methods will be used to account for potential confounding. The propensity to receive surgical counselling will be estimated using logistic regression including potential confounders such as duration and effectiveness of prior DA treatment, the amount and severity of side effects to DA treatment measured at study entrance measured with the PROCTCAE, age at inclusion, gender (male or female), type of centre treatment takes place (secondary or tertiary), physician enrolling the patient (endocrinologist, gynaecologist, or neurosurgeon), prolactinoma size (micro- or macroadenoma), patient and MDT treatment preference pre- and post-counselling (neutral, medication, or surgery), and the likelihood of positive surgical outcome (unlikely, possibly, or likely) as estimated post hoc by two neurosurgeons blinded to received treatment. Adequacy of the propensity model will be assessed by checking if standardised mean differences after propensity adjustment are below 0.10 and by inspecting balancing plots.

Inverse probability weighting based on the propensity score will be used to balance the treatment groups. After observations are reweighted, treatment groups are 
compared in the same way as in the RCTs. Propensity matching will be used as a sensitivity analysis.

\section{Statistical analyses of secondary outcomes}

We will perform several additional analyses of the secondary parameters. When appropriate, we will perform $t$-tests for continuous variables, or regression analysis with adjustment for prognostic baseline variables to increase efficiency and account for suspected confounding. For continuous variables (disease burden, and depression and anxiety scores, for example) measured several times during follow-up and probably missing data of some patients at some time points, longitudinal effects of the treatment strategies will be assessed with linear mixed models. For dichotomous variables, we will calculate differences in risks with a 95\% confidence interval and compare the two treatment arms with the use of a chisquare test. Repeated measurements of dichotomous variables will be analysed with generalised estimating equations (if needed with reweighting for missing data) and logistic regression mixed models.

Combining the results of the different trials Since the design of the three RCTs is similar, with the main difference being the duration of DA pre-treatment, the main results will be compared between the three RCTs and the PRolaCT-O study. We first compare the baseline characteristics between the studies. Then, a combined analysis including PRolaCT-O and the RCTs will be performed, using meta-analysis methods. If the results of the different studies differ substantially, we will explore if the differences can be explained by different patient populations in the different studies, using individual patient data (IPD) meta-regression methods, combined with inverse probability weighting.

Moreover, we will compare surgical outcomes between the intervention groups of the three RCTs, using a multivariate analysis, to compare the effects of the duration of DA pre-treatment on surgical outcomes.

In addition to the aforementioned analyses that will be performed according to the intention-to-treat principle, we will perform per-protocol analyses for both our primary and secondary endpoints.

\section{Economic analysis}

\section{Cost-effectiveness analysis (CEA)}

The economic evaluation will consist of both trial-based cost-effectiveness analyses for all three RCTs and PRolaCT-O (costs per additional patient in remission and cost per quality-of-life adjusted life-year (QALY)) using observed cost and outcome data, and a modelbased cost-utility analysis with a lifetime horizon (costs per QALY), in which all different treatment options are compared. In the trial-based economic evaluations, the effects of surgical treatment for patients with prolactinomas will be compared to standard care and related to the difference in costs. Differences in mean costs and effects between strategies will be compared with two-sided bootstrapping. In a net-benefit analysis, costs will be related to the outcomes and presented in a costeffectiveness acceptability curve. No discounting will be applied due to the short time horizon of the economic evaluation. The evaluation will be performed from a societal perspective. Sensitivity analyses will be carried out for the most important assumptions.

In the model-based cost-utility analysis, a decision tree model will be used to extrapolate the trial results to lifetime costs and QALYs for different surgical treatment options (with and without pre-treatment with cabergoline) in comparison with usual care [29, 30]. In this lifetime cost-utility analysis, costs will be discounted at a percentage of $4 \%$ and effects at a percentage of $1.5 \%$, according to the Dutch guidelines for health economic research [34]. Sensitivity analysis will be carried out for the most important input parameters.

Health care use and absence from work will be assessed with the iMCQ and iPCQ. For the valuation of health care, standard prices published in the Dutch costing guidelines will be used [35]. Costs of absenteeism from paid work will be calculated using the friction cost method. QALYs will be estimated with aid of the EQ-5D-5L. Utilities will be calculated from the EQ-5D-5L questionnaire using the Dutch tariff [36]. Using the area-under-the-curve method for the utility scores obtained for each participant, the QALY outcome per participant will be obtained for the trialbased cost-effectiveness analysis. Furthermore, utility values will be used as input in the model for the lifetime cost-effectiveness analysis.

\section{Data collection}

During the study, all study data will be collected and stored in the data management system Castor EDC, with the use of an electronic case record form (eCRF), secured with personal login codes. Participants will be assigned a unique subject number. All data will be collected, stored, and analysed in a coded manner and in accordance with the General Data Protection Regulation (GDPR). The key to subjects' identification codes will be stored securely at the study site, separate from all other study-related data. After the study ends, all exports of the study data and eCRFs will be stored digitally on the hospital server of the LUMC in a protected folder with access limited to the principal and coordinating investigators of the LUMC. Study data will be stored for 10 years after the study ends. There will be no collection or storing of biological specimens for this study. 


\section{Safety reporting}

For the RCTs, any adverse event (AE) that may occur in the standard care groups or the intervention groups after patients are referred back to their own hospital is either an expected result from standard care (and thus measured as part of the study outcomes) or not at all related to the prolactinoma or the received treatment. It is thus highly unlikely that any $\mathrm{AE}$ occurring in these patients is related to study participation. AEs will therefore only be recorded for participants in the intervention groups, when they are treated and under medical follow-up in the participating neurosurgical expertise centres. Furthermore, all observed or patient-reported adverse events will be recorded, unless there is a clear relation to pregnancy, e.g. nausea due to morning sickness. All AEs will be followed until they have abated, or until a stable situation has been reached.

All AEs are part of the safety data discussed with the Data Safety Monitoring Board. Moreover, the MREC is notified of all AEs that either result in death, are lifethreatening, require non-elective hospitalisation or prolongation of hospitalisation to a maximum of 10 days in case of hospitalisation for prolactinoma surgery, result in persistent or significant disability or incapacity, or are a congenital anomaly or birth defect.

AEs that may occur in participants in PRolaCT-O will not be recorded, because the observational nature of their participation will imply that any AE will not be related to study participation.

\section{Monitoring}

\section{Data Safety Monitoring Board (DSMB)}

An independent DSMB will perform ongoing safety surveillance for the RCTs. The main responsibility of the DSMB for this study is guarding participant safety. Although complications related to the intervention are uncommon, this is the main interest for the DSMB. No formal interim analyses will be performed.

Monitoring of trial conduct and integrity will be executed every 6 months by internal monitors of the LUMC.

\section{Ethics and dissemination}

The research protocol for the PRolaCT study has been reviewed and approved by the Medical Research Ethics Committee (MREC) of the LUMC and, when appropriate, by the local Institutional Research Boards (IRBs) of all participating centres. There is no formal steering committee or endpoint adjudication committee. However, there is an advisory board consisting of the principal investigators from St. Antonius Ziekenhuis, Medisch Spectrum Twente, and Radboud University Medical Centre, and our patient representative, chair of the
Dutch Pituitary Foundation. The advisory board has been consulted during the development phase of the study protocol and is consulted for important protocol modifications. In addition, important protocol modifications are discussed with the DSMB and reviewed by the MREC of the LUMC, as is required by Dutch law on Medical Research involving Human Participants. After MREC approval, all principal investigators, and when deemed appropriate by the MREC, all participants, and the local IRBs will be notified, and the trial registry will be updated. Important protocol modifications will be declared transparently in the publication of the primary results.

Standard of care, which is primarily assessed in this study, is being covered by regular patient insurance. Damages to research participants through injury or death caused by the study will be reimbursed through a standard insurance for research participants, which covers the damage that becomes apparent during the study, or within 4 years after the end of the study. The risk of injury or death due to study interventions, which is neurosurgical counselling, is virtually non-existent.

After completion of the trials, the primary results of the RCTs will be unreservedly published in an open access and peer-reviewed journal.

\section{Discussion}

\section{Rationale for the study design}

Prolonged DA treatment for prolactinoma, which is current standard practice $[1,4,5]$, is very effective in controlling hyperprolactinaemia and restoring gonadal function. However, patients may suffer from substantial side effects compromising HRQoL to a greater extent than previously described, while remission rates following DA treatment are lower than previously thought [5$13,18-22,37]$. Although there is a low risk of complications, surgical resection induces swift disease remission in most patients, which may allow a drug-free future [5, $17,23-26,28,38-46]$ with improved or even normalised HRQoL, and may thus deserve a more prominent place in the treatment of prolactinoma patients. However, loss of pituitary function and life-threatening complications must be considered.

The PRolaCT studies combine three randomised clinical trials and an observational cohort and are designed to compare both treatment strategies for patientrelevant outcomes, such as disease remission and HRQoL. For the RCT parts of PRolaCT, patients consent to randomisation between surgical counselling or standard care. If we would randomise between surgery versus DA treatment, all patients would have had to be referred to a neurosurgical expertise centre for surgical counselling prior to entering the study. With our pragmatic RCT design, only patients who randomise for surgical 
counselling are referred to the regional MDT to receive detailed information about surgical treatment for prolactinoma and standard care is delivered in the regional hospital. Patients who randomise for standard care are thus not burdened with referral to a neurosurgical expertise centre and neurosurgical counselling. Although a greater appeal on regional networks is made, the pragmatic design of PRolaCT intends to lower the threshold for randomisation.

The study intervention in PRolaCT is thus the neurosurgical counselling rather than surgical intervention, which allows patients in the intervention groups to opt for surgery. If there is a substantial proportion of patients in the intervention group who do not undergo surgery, it would lead to an attenuation of found effects. Furthermore, as a part of standard care, participants in the control groups may still undergo surgery in the follow-up of the study (which is sporadically needed in case of severe DA intolerance or resistance). The expected cross-over between treatments was taken into account for the sample size calculation, resulting in a larger sample size needed. However, we anticipate that neurosurgical counselling greatly contributes to patients' decision for a specific treatment, and therefore, our approach may decrease the risk of study drop-out due to disappointment over the outcome of randomisation.

\section{Strong patient preference}

In our experience, many prolactinoma patients are willing to undergo surgery, which was confirmed by a recent survey conducted by the Dutch Pituitary Foundation. Nonetheless, patients' willingness to be randomised appears diminished due to a strong preference for a specific treatment, which was generally, but not exclusively, for surgical resection, and patient recruitment has thus far been limited. To overcome this impaired patient recruitment, PRolaCT-O primarily aims to compare HRQoL, remission rates, and cost-effectiveness of neurosurgical and medical prolactinoma treatment in patients who are eligible to participate in one of the RCTs, but refuse randomisation.

The non-interventional nature of PRolaCT-O has some limitations, mainly based on the assumption that all confounders are known and adequately measured. For PRolaCT-O, confounding by indication can be expected, but comprehensive baseline measurements, including exploration of patients' and physicians' motivation for a specific treatment choice, may allow for correction for expected confounders, such as the expected likelihood of complete resection, the effectiveness of previous DA treatment, or the amount and severity of side effects to DA treatment at study baseline. Nonetheless, reviews comparing observational studies and RCTs have found effect estimate differences, although not all statistically significant, even when correction for confounding by propensity scoring was used $[47,48]$. However, these differences may as well be explained by varying outcome definitions, differentially distributed effect modifiers in the underlying population, when estimates of population-averaged effects are used, and bias in both RCTs and observational studies. Therefore, found differences between effect estimates of observational and randomised studies do not necessarily mean observational studies are less reliable [49]. Clearly, PRolaCT-O cannot replace the randomised studies that are and will remain the mainstay of PRolaCT. However, PRolaCT-O, which aims to have a lower patient threshold for participation, might be more easily implemented in clinical practice, and we believe will provide study results earlier than the RCTs.

Collection of "real-world" data with PRolaCT-O might provide interesting insights in patient characteristics (e.g. presence and severity of symptoms or side effects, prolactinoma characteristics), which may be used for clinical decision-making, facilitating more individually tailored practice of evidence-based medicine.

\section{Trial status}

Current protocol version 5, issue date 7 Jan 2021. Recruitment started June 21, 2019, and is planned to be completed in April 2022.

\section{Abbreviations \\ CEA: Cost-effectiveness analysis; DA: Dopamine agonist; DSMB: Data safety monitoring board; eCRF: Electronic case record form; eGFR: Glomerular filtration rate; EDC: Electronic data capture; GDPR: General data protection regulation; HADS: Hospital anxiety and depression scale; HRQoL: Health- related quality of life; ICD: Impulse control disorder; ICD-Q: Impulse control disorder questionnaire; IPD: Individual patient data; IRB: Institutional research board; LBNQ: Leiden bother and needs questionnaire; MCQ: Medical consumption questionnaire; MHS: Mental health scale; MREC: Medical research ethics committee; MRI: Magnetic resonance imaging; PCQ: Productivity cost questionnaire; PRO-CTCAE: Patient-reported outcomes version of the common terminology criteria for adverse events; \\ PROM: Patient-reported outcome measurement; QALY: Quality-adjusted life years; RCT: Randomised clinical trial; SF-36: Short-Form Health Survey; SPIRIT: Standard protocol items: recommendations for interventional trials}

\section{Supplementary Information}

The online version contains supplementary material available at https://doi. org/10.1186/s13063-021-05604-y.

Additional file 1. SPIRIT checklist

Additional file 2. First MERC approval protocol dated 12 March 2019 (original Dutch)

Additional file 3. First MERC approval protocol dated 12 March 2019 (English translation)

Additional file 4. MERC approval amendment PRolaCT-O dated 21 January 2020 (original Dutch)

Additional file 5. MERC approval amendment PRolaCT-O dated 21 January 2020 (English translation)

Additional file 6. Grant ZonMw dated 29 June 2017 (original Dutch)

Additional file 7. Grant ZonMw dated 29 June 2017 (English translation) 


\section{Acknowledgements}

The authors thank Daniel Lobatto for his contributions to the start of PRolaCT. The authors also thank the co-investigators of the Dutch Prolactinoma Study Group: Maarten van Aken, HagaZiekenhuis; Carolien Boot, LangeLand Ziekenhuis; Carmen F.A. Eustatia-Rutten, Alrijne ziekenhuis; Ronald Groote Veldman, Medisch Spectrum Twente; William van Houtum, Spaarne Gasthuis; C.A.H. (Ineke) Janssen, Groene Hart Ziekenhuis; Nienke KeldermanKolk, Alrijne Ziekenhuis; Simon W. Kok, Alrijne Ziekenhuis; J.A.A. (Ton) Meijer, Albert Schweizer Ziekenhuis; Sharita R. Ramautar, Haaglanden Medisch Centrum; Lisanne C.C.J. van der Plas-Smans, HagaZiekenhuis; Yu Lan Soei, Sint Franciscus Vlietland Groep; Sanne Swinnen, Rijnstate; Sjoerd W. van Thiel, Amphia; Marieke F.G. Verberg, Medisch Spectrum Twente \& Ziekenhuis Groep Twente; Titia M. Vriesendorp, Isala; and Iris M.E. Wentholt, Onze Lieve Vrouwe Gasthuis.

\section{Authors' contributions}

NRB, WRB, AHZN, and IMZ conceived of the study. IMZ and ICMP initiate and implement the study design. WRF and NRB are grant holders. OMD and SC provided statistical expertise in clinical trial design and IMZ will be conducting the primary statistical analysis. All authors contributed to the refinement of the study protocol and approved the final manuscript, no professional writers have been involved.

\section{Funding}

ZonMw (semi-government), grant ID: 843002806. The funding source had no role in the design of this study and will not have any role during its execution, analyses, interpretation of the data, or decision to submit results.

\section{Availability of data and materials}

The complete trial dataset will be stored by and accessible to the coordinating centre (LUMC). All principal investigators from the participating centres will have access to datasets containing only participants from their own centres. Analysed datasets will be available from the corresponding author upon reasonable request.

\section{Declarations}

\section{Ethics approval and consent to participate}

Ethical approval has been obtained from the Medical Ethics Review Committee Leiden-The Hague-Delft (P18.219, 12 March 2019). Written informed consent to participate will be obtained from all participants.

\section{Consent for publication}

Not applicable.

\section{Competing interests}

The authors declare that they have no competing interests.

\section{Author details}

${ }^{1}$ Department of Neurosurgery, Leiden University Medical Center, Albinusdreef 2, 2333ZA Leiden, The Netherlands. ${ }^{2}$ Department of Medicine, Division of Endocrinology, Leiden University Medical Center, Albinusdreef 2, 2333ZA Leiden, The Netherlands. ${ }^{3}$ Department of Medicine, Center for Endocrine Tumours Leiden, Leiden University Medical Center, Albinusdreef 2, 2333ZA Leiden, The Netherlands. ${ }^{4}$ Department of Biomedical Data Sciences, Section Medical Decision Making, Leiden University Medical Center, Albinusdreef 2, 2333ZA Leiden, The Netherlands. ${ }^{5}$ Department of Medicine, Division of Endocrinology, Amsterdam University Medical Center - location AMC, Meibergdreef 9, 1105AZ Amsterdam, The Netherlands. ${ }^{6}$ Department of Neurosurgery, Radboud University Medical Center, Geert Grooteplein Zuid 10, 6525GA Nijmegen, The Netherlands. 'Department of Internal Medicine, Rijnstate, Wagnerlaan 55, 6815AD Arnhem, The Netherlands. ${ }^{8}$ Department of Neurosurgery, Elisabeth-Tweesteden Ziekenhuis, Hilvarenbeekseweg 60, 5022GC Tilburg, The Netherlands. 'Department of Clinical Epidemiology, Leiden University Medical Center, Albinusdreef 2, 2333ZA Leiden, The Netherlands. ${ }^{10}$ Department of Medicine, Division of Endocrinology, Amsterdam University Medical Center - location VUmc, De Boelelaan 1118, $1081 \mathrm{HZ}$ Amsterdam, The Netherlands. ${ }^{11}$ Department of Medicine, Division of Endocrinology, Erasmus Medical Center, Doctor Molewaterplein 40, 3015GD Rotterdam, The Netherlands. ${ }^{12}$ Dutch Pituitary Foundation, PO BOX 1014, 3860BA Nijkerk, The Netherlands. ${ }^{13}$ Department of Neurosurgery, Amsterdam
University Medical Center - location AMC, Meibergdreef 9, 1105AZ Amsterdam, The Netherlands. ${ }^{14}$ Department of Gynaecology, Reinier de Graaf Gasthuis, Reinier de Graafweg 5, 2625AD Delft, The Netherlands. ${ }^{15}$ Department of Medicine, Division of Endocrinology, University Medical Center Groningen, Hanzeplein 1, 9713GZ Groningen, The Netherlands. ${ }^{16}$ Department of Internal Medicine, Elisabeth-Tweesteden Ziekenhuis, Hilvarenbeekseweg 60, 5022 GC Tilburg, The Netherlands. ${ }^{17}$ Department of Medicine, Division of Endocrinology, University Medical Center Utrecht, Heidelberglaan 100, 3584CX Utrecht, The Netherlands. ${ }^{18}$ Department of Medicine, Division of Endocrinology, Radboud University Medical Center, Geert Grooteplein Zuid 10, 6525GA Nijmegen, The Netherlands.

${ }^{19}$ Department of Internal Medicine, St. Antonius Ziekenhuis, Koekoekslaan 1, 3435CM, Nieuwegein, The Netherlands.

Received: 11 November 2020 Accepted: 5 September 2021 Published online: 25 September 2021

\section{References}

1. Molitch ME. Diagnosis and treatment of pituitary adenomas: a review. Jama. 2017;317(5):516-24. https://doi.org/10.1001/jama.2016.19699.

2. Gruppetta M, Mercieca C, Vassallo J. Prevalence and incidence of pituitary adenomas: a population based study in Malta. Pituitary. 2013;16(4):545-53. https://doi.org/10.1007/s11102-012-0454-0.

3. Kars M, Souverein PC, Herings RM, Romijn JA, Vandenbroucke JP, de Boer A, et al. Estimated age- and sex-specific incidence and prevalence of dopamine agonist-treated hyperprolactinemia. J Clin Endocrinol Metab. 2009;94(8):2729-34. https://doi.org/10.1210/jc.2009-0177.

4. Melmed S, Casanueva FF, Hoffman AR, Kleinberg DL, Montori VM, Schlechte JA, et al. Diagnosis and treatment of hyperprolactinemia: an Endocrine Society clinical practice guideline. J Clin Endocrinol Metab. 2011;96(2):27388. https://doi.org/10.1210/jc.2010-1692.

5. Zamanipoor Najafabadi AH, Zandbergen IM, de Vries F, LHA B, van den Akker-van Marle ME, Pereira AM, et al. Surgery as a viable alternative firstline treatment for prolactinoma patients. A systematic review and metaanalysis. J Clin Endocrinol Metab. 2020;105(3):e32-41. https://doi.org/10.121 0/clinem/dgz144.

6. Andela CD, Niemeijer ND, Scharloo M, Tiemensma J, Kanagasabapathy S, Pereira AM, et al. Towards a better quality of life (QoL) for patients with pituitary diseases: results from a focus group study exploring QoL. Pituitary. 2015;18(1):86-100. https://doi.org/10.1007/s11102-014-0561-1.

7. Elenkova A, Shabani R, Kalinov K, Zacharieva S. Increased prevalence of subclinical cardiac valve fibrosis in patients with prolactinomas on longterm bromocriptine and cabergoline treatment. Eur J Endocrinol. 2012; 167(1):17-25. https://doi.org/10.1530/EJE-12-0121.

8. Ferrari C, Paracchi A, Mattei AM, de Vincentiis S, D'Alberton A, Crosignani P. Cabergoline in the long-term therapy of hyperprolactinemic disorders. Acta Endocrinol. 1992;126(6):489-94. https://doi.org/10.1530/acta.0.1260489.

9. Merola B, Sarnacchiaro F, Colao A, Di Somma C, Di Sarno A, Landi ML, et al. CV 205-502 in the treatment of tumoral and non-tumoral hyperprolactinemic states. Biomed Pharmacother. 1994;48(3-4):167-74.

10. Vallette S, Serri K, Rivera J, Santagata P, Delorme S, Garfield N, et al. Longterm cabergoline therapy is not associated with valvular heart disease in patients with prolactinomas. Pituitary. 2009;12(3):153-7. https://doi.org/10.1 007/s11102-008-0134-2.

11. Webster J, Piscitelli G, Polli A, D'Alberton A, Falsetti L, Ferrari C, et al. The efficacy and tolerability of long-term cabergoline therapy in hyperprolactinaemic disorders: an open, uncontrolled, multicentre study. European Multicentre Cabergoline Study Group. Clin Endocrinol. 1993;39(3): 323-9. https://doi.org/10.1111/j.1365-2265.1993.tb02372.x.

12. Webster J, Piscitelli G, Polli A, Ferrari Cl, Ismail I, Scanlon MF. A comparison of cabergoline and bromocriptine in the treatment of hyperprolactinemic amenorrhea. Cabergoline Comparative Study Group. New Engl J Med. 1994; 331(14):904-9. https://doi.org/10.1056/NEJM199410063311403.

13. De Sousa SM, Chapman IM, Falhammar H, Torpy DJ. Dopa-testotoxicosis: disruptive hypersexuality in hypogonadal men with prolactinomas treated with dopamine agonists. Endocrine. 2017;55(2):618-24. https://doi.org/10.1 007/s12020-016-1088-1.

14. Bancos I, Nannenga MR, Bostwick JM, Silber MH, Erickson D, Nippoldt TB. Impulse control disorders in patients with dopamine agonist-treated prolactinomas and nonfunctioning pituitary adenomas: a case-control study. Clin Endocrinol. 2014;80(6):863-8. https://doi.org/10.1111/cen.12375. 
15. Barake M, Evins AE, Stoeckel L, Pachas GN, Nachtigall LB, Miller KK, et al. Investigation of impulsivity in patients on dopamine agonist therapy for hyperprolactinemia: a pilot study. Pituitary. 2014;17(2):150-6. https://doi. org/10.1007/s11102-013-0480-6

16. Moore TJ, Glenmullen J, Mattison DR. Reports of pathological gambling, hypersexuality, and compulsive shopping associated with dopamine receptor agonist drugs. JAMA Intern Med. 2014;174(12):1930-3. https://doi org/10.1001/jamainternmed.2014.5262.

17. Tampourlou M, Trifanescu R, Paluzzi A, Ahmed SK, Karavitaki N. Therapy of endocrine disease: surgery in microprolactinomas: effectiveness and risks based on contemporary literature. Eur J Endocrinol. 2016;175(3):R89-96. https://doi.org/10.1530/EJE-16-0087

18. Cesar de Oliveira Naliato E, Dutra Violante AH, Caldas D, Lamounier Filho A, Rezende Loureiro C, Fontes R, et al. Quality of life in women with microprolactinoma treated with dopamine agonists. Pituitary. 2008;11(3): 247-54. https://doi.org/10.1007/s11102-008-0091-9.

19. Johnson MD, Woodburn CJ, Vance ML. Quality of life in patients with a pituitary adenoma. Pituitary. 2003;6(2):81-7. https://doi.org/10.1023/B:PITU 0000004798.27230.ed.

20. Kars M, van der Klaauw AA, Onstein CS, Pereira AM, Romijn JA. Quality of life is decreased in female patients treated for microprolactinoma. Eur J Endocrinol. 2007;157(2):133-9. https://doi.org/10.1530/EJE-07-0259.

21. Ritvonen E, Karppinen A, Sintonen H, Vehkavaara S, Kivipelto L, Roine RP, et al. Normal long-term health-related quality of life can be achieved in patients with functional pituitary adenomas having surgery as primary treatment. Clin Endocrinol. 2015;82(3):412-21. https://doi.org/10.1111/cen.12 550.

22. Andela CD, Scharloo M, Pereira AM, Kaptein AA, Biermasz NR. Quality of life (QoL) impairments in patients with a pituitary adenoma: a systematic review of QoL studies. Pituitary. 2015;18(5):752-76. https://doi.org/10.1007/ s11102-015-0636-7.

23. Dallapiazza RF, Jane JA Jr. Outcomes of endoscopic transsphenoidal pituitary surgery. Endocrinol Metab Clin North Am. 2015;44(1):105-15. https://doi.org/10.1016/j.ecl.2014.10.010.

24. Tabaee A, Anand VK, Barron Y, Hiltzik DH, Brown SM, Kacker A, et al. Predictors of short-term outcomes following endoscopic pituitary surgery. Clin Neurol Neurosurg. 2009;111(2):119-22. https://doi.org/10.1016/j. clineuro.2008.09.010

25. Wang F, Zhou T, Wei S, Meng X, Zhang J, Hou Y, et al. Endoscopic endonasal transsphenoidal surgery of 1,166 pituitary adenomas. Surg Endosc. 2015;29(6):1270-80. https://doi.org/10.1007/s00464-014-3815-0.

26. Paluzzi A, Fernandez-Miranda JC, Tonya Stefko S, Challinor S, Snyderman CH, Gardner PA. Endoscopic endonasal approach for pituitary adenomas: a series of 555 patients. Pituitary. 2014;17(4):307-19. https://doi.org/10.1007/ s11102-013-0502-4.

27. Casanueva FF, Barkan AL, Buchfelder M, Klibanski A, Laws ER, Loeffler JS, et al. Criteria for the definition of Pituitary Tumor Centers of Excellence (PTCOE): a Pituitary Society statement. Pituitary. 2017;20(5):489-98. https:// doi.org/10.1007/s11102-017-0838-2.

28. Dehdashti AR, Ganna A, Karabatsou K, Gentili F. Pure endoscopic endonasal approach for pituitary adenomas: early surgical results in 200 patients and comparison with previous microsurgical series. Neurosurgery. 2008;62(5): 1006-15; discussion 15-7. https://doi.org/10.1227/01.neu.0000325862.83 961.12.

29. Jethwa PR, Patel TD, Hajart AF, Eloy JA, Couldwell WT, Liu JK. Costeffectiveness analysis of microscopic and endoscopic transsphenoidal surgery versus medical therapy in the management of microprolactinoma in the United States. World Neurosurg. 2016;87:65-76. https://doi.org/10.101 6/j.wneu.2015.10.090.

30. Zygourakis CC, Imber BS, Chen R, Han SJ, Blevins L, Molinaro A, et al. Costeffectiveness analysis of surgical versus medical treatment of prolactinomas. J Neuro Surg B Skull Base. 2017;78(2):125-31.

31. Aaronson NK, Muller M, Cohen PD, Essink-Bot ML, Fekkes M, Sanderman R, et al. Translation, validation, and norming of the Dutch language version of the SF-36 Health Survey in community and chronic disease populations. J Clin Epidemiol. 1998:51(11):1055-68. https://doi.org/10.1016/S0895-43 56(98)00097-3.

32. McHorney CA, Ware JE, Jr., Raczek AE. The MOS 36-Item Short-Form Health Survey (SF-36): II. Psychometric and clinical tests of validity in measuring physical and mental health constructs. Med Care. 1993;31(3):247-263, DOI: https://doi.org/10.1097/00005650-199303000-00006.
33. Ware JE, Jr., Sherbourne CD. The MOS 36-item short-form health survey (SF36). I. Conceptual framework and item selection. Med Care. 1992;30(6):473483, DOI: https://doi.org/10.1097/00005650-199206000-00002.

34. Nederland Z. Richtlijn voor het uitvoeren van economische evaluaties in de gezondheidszorg. 2016.

35. Kanters TA, Bouwmans CAM, van der Linden N, Tan SS, Hakkaart-van RL. Update of the Dutch manual for costing studies in health care. PLoS One. 2017;12(11):e0187477. https://doi.org/10.1371/journal.pone.0187477.

36. Versteegh MM, Vermeulen KM, Evers SMAA, de Wit GA, Prenger R, Stolk EA. Dutch Tariff for the five-level version of EQ-5D. Value Health. 2016;19(4):34352. https://doi.org/10.1016/j.jval.2016.01.003

37. Sala E, Bellaviti Buttoni P, Malchiodi E, Verrua E, Carosi G, Profka E, et al. Recurrence of hyperprolactinemia following dopamine agonist withdrawal and possible predictive factors of recurrence in prolactinomas. J Endocrinol Invest. 2016;39(12):1377-82. https://doi.org/10.1007/s40618-016-0483-z.

38. Akin S, Isikay I, Soylemezoglu F, Yucel T, Gurlek A, Berker M. Reasons and results of endoscopic surgery for prolactinomas: 142 surgical cases. Acta Neurochir (Wien). 2016;158(5):933-42. https://doi.org/10.1007/s00701-016-2 $762-z$.

39. D'Haens J, Van Rompaey K, Stadnik T, Haentjens P, Poppe K, Velkeniers B. Fully endoscopic transsphenoidal surgery for functioning pituitary adenomas: a retrospective comparison with traditional transsphenoidal microsurgery in the same institution. Surg Neurol. 2009;72(4):336-40. https://doi.org/10.1016/j.surneu.2009.04.012.

40. Gondim JA, Schops M, de Almeida JP, de Albuquerque LA, Gomes E, Ferraz T, et al. Endoscopic endonasal transsphenoidal surgery: surgical results of 228 pituitary adenomas treated in a pituitary center. Pituitary. 2010;13(1):6877. https://doi.org/10.1007/s11102-009-0195-x.

41. Hofstetter CP, Shin BJ, Mubita L, Huang C, Anand VK, Boockvar JA, et al. Endoscopic endonasal transsphenoidal surgery for functional pituitary adenomas. Neurosurg Focus. 2011;30(4):E10. https://doi.org/10.3171/2011.1. FOCUS10317.

42. Maric A, Kruljac I, Cerina V, Pecina HI, Sulentic P, Vrkljan M. Endocrinological outcomes of pure endoscopic transsphenoidal surgery: a Croatian Referral Pituitary Center experience. Croat Med J. 2012;53(3):224-33. https://doi.org/1 0.3325/cmj.2012.53.224.

43. Smith TR, Hulou MM, Huang KT, Gokoglu A, Cote DJ, Woodmansee WW, et al. Current indications for the surgical treatment of prolactinomas. J Clin Neurosci. 2015;22(11):1785-91. https://doi.org/10.1016/j.jocn.2015.06.001.

44. Tanei T, Nagatani T, Nakahara N, Watanabe T, Nishihata T, Nielsen ML, et al. Use of high-field intraoperative magnetic resonance imaging during endoscopic transsphenoidal surgery for functioning pituitary microadenomas and small adenomas located in the intrasellar region. Neurol Med Chir (Tokyo). 2013;53(7):501-10. https://doi.org/10.2176/nmc.53. 501.

45. Yano S, Kawano T, Kudo M, Makino K, Nakamura H, Kai Y, et al. Endoscopic endonasal transsphenoidal approach through the bilateral nostrils for pituitary adenomas. Neurol Med Chir (Tokyo). 2009;49(1):1-7. https://doi. org/10.2176/nmc.49.1.

46. Frank G, Pasquini E, Farneti G, Mazzatenta D, Sciarretta V, Grasso V, et al. The endoscopic versus the traditional approach in pituitary surgery. Neuroendocrinology. 2006;83(3-4):240-8. https://doi.org/10.1159/000095534.

47. Anglemyer A, Horvath HT, Bero L. Healthcare outcomes assessed with observational study designs compared with those assessed in randomized trials. Cochrane Database Syst Rev. 2014;4(4). https://doi.org/10.1002/14651 858.MR000034.pub2.

48. Hemkens LG, Contopoulos-loannidis DG, loannidis JPA. Agreement of treatment effects for mortality from routinely collected data and subsequent randomized trials: meta-epidemiological survey. BMJ. 2016;352: i493. https://doi.org/10.1136/bmj.i493.

49. Forbes SP, Dahabreh IJ. Benchmarking observational analyses against randomized trials: a review of studies assessing propensity score methods. J Gen Intern Med. 2020;35(5):1396-404. https://doi.org/10.1007/s1 1606-02005713-5.

\section{Publisher's Note}

Springer Nature remains neutral with regard to jurisdictional claims in published maps and institutional affiliations. 